\title{
Perioperative outcomes following reoperation for recurrent insular gliomas
}

\author{
Ramin A. Morshed, MD, ${ }^{1}$ Jacob S. Young, MD, ${ }^{1}$ Seunggu J. Han, MD, ${ }^{2}$ \\ Shawn L. Hervey-Jumper, MD, ${ }^{1}$ and Mitchel S. Berger, MD ${ }^{1}$ \\ 'Department of Neurological Surgery, University of California, San Francisco, California; and 2Department of Neurological \\ Surgery, Oregon Health \& Science University, Portland, Oregon
}

\begin{abstract}
OBJECTIVE Greater extent of resection (EOR) improves overall survival and progression-free survival for patients with low- and high-grade glioma. While resection for newly diagnosed insular gliomas can be performed with minimal morbidity, perioperative morbidity is not clearly defined for patients undergoing a repeat resection for recurrent insular gliomas. In this study the authors report on tumor characteristics, tumor EOR, and functional outcomes in patients undergoing reoperation for recurrent insular glioma.
\end{abstract}

METHODS Adult patients with insular gliomas (WHO grades II-IV) who underwent index resection followed by reoperation were identified through the University of California San Francisco Brain Tumor Center. Treatment history and functional outcomes were collected retrospectively from the electronic medical record. Pre- and postoperative tumor volumes were quantified using software with region-of-interest analysis based on FLAIR and T1-weighted postgadolinium sequences from pre- and postoperative MRI.

RESULTS Forty-four patients ( $63.6 \%$ male, $36.4 \%$ female) undergoing 49 reoperations for recurrent insular tumors were identified with a median follow-up of 741 days. Left- and right-sided tumors comprised $52.3 \%$ and $47.7 \%$ of the cohort, respectively. WHO grade II, III, and IV gliomas comprised $46.9 \%, 28.6 \%$, and $24.5 \%$ of the cohort, respectively. Ninetyfive percent (95.9\%) of cases involved language and/or motor mapping. Median EOR of the insular component of grade II, III, and IV tumors were $82.1 \%, 75.0 \%$, and $94.6 \%$, respectively. EOR during reoperation was not impacted by BergerSanai insular zone or tumor side. At the time of reoperation, $44.9 \%$ of tumors demonstrated malignant transformation to a higher WHO grade. Ninety-day postoperative assessment confirmed that $91.5 \%$ of patients had no new postoperative deficit attributable to surgery. Of those with new deficits, $3(6.4 \%)$ had a visual field cut and $1(2.1 \%)$ had hemiparesis (strength grade 1-2/5). The presence of a new postoperative deficit did not vary with EOR.

CONCLUSIONS Recurrent insular gliomas, regardless of insular zone and pathology, may be reoperated on with an overall acceptable degree of resection and safety despite their anatomical and functional complexities. The use of intraoperative mapping utilizing asleep or awake methods may reduce morbidity to acceptable rates despite prior surgery.

https://thejns.org/doi/abs/10.3171/2018.4.JNS18375

KEYWORDS insula; glioma; reoperation; oncology

$\mathrm{I}$ NSULAR gliomas remain challenging lesions to address surgically. Anatomically, nearby medial and lateral lenticulostriate and insular arteries supply surrounding functional language and motor pathways and are at risk of injury if not identified and preserved. Yet, with the use of functional mapping and microsurgical techniques, these insular gliomas are accessible and surgically resectable with low rates of morbidity., $3,5,8,10,13,19$ It is well established that greater extent of resection (EOR) improves overall and progression-free survival as well as seizure control for patients with newly diagnosed low- and high-grade insular glioma. ${ }^{5,711,13}$ Similarly, repeat resection of gliomas has also been shown to significantly improve these same outcomes for patients with recurrent low- and high-grade gliomas. ${ }^{2,12,16-18}$

Although outcomes after resection for newly diagnosed insular gliomas have been previously reported, perioperative morbidity for patients undergoing a repeat resection

ABBREVIATIONS DVT = deep vein thrombosis; DWI = diffusion-weighted imaging; EOR = extent of resection; MCA = middle cerebral artery; MRC = Medical Research Council; RT = radiation therapy; UCSF = University of California, San Francisco.

SUBMITTED February 8, 2018. ACCEPTED April 9, 2018.

INCLUDE WHEN CITING Published online September 21, 2018; DOI: 10.3171/2018.4.JNS18375. 
for a recurrent insular glioma is understudied and not separately reported. In this paper we report on the EOR, morbidity rates, and technical challenges that arose in a cohort of patients undergoing reoperation for recurrent insular gliomas.

\section{Methods}

\section{Patient Selection and Characteristics}

After obtaining approval from the IRB of the University of California, San Francisco (UCSF), a search query was performed through the UCSF tumor registry to include patients with a pathologic diagnosis of a glioma (WHO grades II-IV) located within the insular region who underwent an index resection followed by reoperation. Patients were excluded if their glioma involved any part of the insula but resection of the insular component of the tumor was not undertaken during reoperation. Fortyfour patients with operations between 2004 and 2017 met criteria for inclusion. The majority of patients had their index surgery performed at UCSF (42 of the 44 patients). Patient and tumor characteristics in addition to perioperative outcomes were collected retrospectively from operative, radiology, pathology, and scanned documents available through the UCSF electronic medical record. Consistent with prior reports, the Berger-Sanai insular glioma classification system was used to identify the location of each tumor based on preoperative FLAIR or T1-weighted postgadolinium scans depending on the tumor grade. ${ }^{1,5,13}$ In brief, the insula was divided into 4 zones, and tumor location was assigned to one or more of these zones. For tumors occupying more than one zone, this condition was denoted as such (e.g., zone I + II). In cases in which the tumor occupied all 4 zones, these insular gliomas were defined as "giant."

\section{Patient Outcome Measurements}

Patients underwent sequential neurological examinations performed by 4 clinicians during the perioperative period (the senior attending neurosurgeon, a neurosurgical resident, a speech and language neurophysiologist, and an attending neurooncologist). Clinical examinations were performed every day during the postoperative period, and at each follow-up appointment by at least 1 of the abovementioned clinicians. Short-term neurological morbidity was defined as new-onset language or sensorimotor deficits by the time of discharge (within 3-5 days of surgery). Long-term neurological morbidity was defined as persistent dysfunction 90 days after surgical intervention. If differences existed between findings of the 4 examiners, the results showing the greatest impairment at a given time point was used. MRI results were reviewed to confirm that the patient's symptoms were not a function of tumor progression at each time point. Patients were excluded from analysis when examining 90-day postoperative neurological deficits if they were lost to follow-up or had new neurological symptoms after discharge related to rapid tumor progression and not attributable to surgery.

\section{Surgical Approach}

After patient positioning, patients were registered for navigation based on preoperative imaging obtained within 24 hours prior to surgery using Brainlab software. During the surgical approach the prior bone flap was used for exposure unless the size or location of recurrent tumor called for enlargement of the surgical field by enlarging the craniotomy. Microsurgical technique was used for tumor removal and intraoperative mapping was used in the majority of cases with patients either asleep or awake based on the clinical need.

\section{Volumetric Analysis and EOR Quantification}

Preoperative and postoperative tumor volumes within the insular component only were quantified using Brainlab SmartBrush software. Manual segmentation was performed with region-of-interest analysis based on FLAIR and T1-weighted postgadolinium sequences from pre- and postoperative MRI scans to quantify insular-specific tumor volume. EOR was calculated as: (preoperative tumor volume - postoperative tumor volume)/preoperative tumor volume $\times 100 \%$. Manual segmentations were performed by 1 operator (R.A.M.) with tumor volumetrics verified for accuracy after an initial training period (S.L.H.J.). Knowledge of clinical outcomes was withheld from all study participants involved in tumor volumetrics and perioperative outcome data collection. Preoperative MR images were obtained within 24 hours prior to resection, and postoperative images were all obtained within 48 hours after resection. To ensure that postoperative FLAIR signal was not surgically induced edema or ischemia, FLAIR pre- and postoperative MR images were carefully compared alongside diffusion-weighted imaging (DWI) sequences prior to including each region in the volume segmentation.

\section{Statistical Analysis}

Descriptive statistics were used to define the patient cohort, tumor locations and characteristics, treatment details, EOR, and neurological outcomes. One-way ANOVA and chi-square tests were performed for univariate analysis. The level of significance was 0.05 for all analyses. Statistical analysis was performed using the JMP Pro statistical program (version 13, SAS Institute).

\section{Results}

\section{Patient and Tumor Characteristics}

Forty-four patients (63.6\% male, $36.4 \%$ female) undergoing 49 reoperations for recurrent insular gliomas were identified and had a median follow-up of 2 years (Table 1). Left- and right-sided tumors comprised $52.3 \%$ and $47.7 \%$ of the cohort, respectively. WHO grade II, III, and IV gliomas comprised $46.9 \%, 28.6 \%$, and $24.5 \%$ of the cohort, respectively. Additionally, $81.6 \%$ had undergone some form of adjuvant therapy (chemotherapy, radiation therapy [RT], or a combination of both) preoperatively. When examining the Berger-Sanai insular zone, 22 tumors were localized to 1 insular zone, 22 were localized to 2 zones, and 5 tumors were classified as involving all 4 zones (i.e., giant) at the time of reoperation. Compared to tumor zone at index surgery, 17 (34.7\%) of recurrent insular gliomas occurred at least in some part within a new insular zone. 
TABLE 1. Patient and tumor characteristics

\begin{tabular}{|c|c|}
\hline Characteristic & Value \\
\hline No. of patients & 44 \\
\hline No. of surgeries & 49 \\
\hline Mean age $\pm S D$, yrs & $41.8 \pm 9.4$ \\
\hline \multicolumn{2}{|l|}{ Sex, $n(\%)$} \\
\hline Male & $28(63.6)$ \\
\hline Female & $16(36.4)$ \\
\hline \multicolumn{2}{|l|}{ Laterality, n (\%) } \\
\hline $\mathrm{Rt}$ & $21(47.7)$ \\
\hline $\mathrm{Lt}$ & $23(52.3)$ \\
\hline \multicolumn{2}{|l|}{ WHO grade, $n(\%)$} \\
\hline II & $23(46.9)$ \\
\hline III & $14(28.6)$ \\
\hline IV & $12(24.5)$ \\
\hline Median time to reop $\pm \mathrm{SD},{ }^{*} \mathrm{yrs}$ & $4.5 \pm 3.0$ \\
\hline Malignant transformation, $\mathrm{n}(\%)$ & $22(44.9)$ \\
\hline \multicolumn{2}{|l|}{ Berger-Sanai zone, $\mathrm{n}(\%)$} \\
\hline 1 & $2(4.1)$ \\
\hline II & $3(6.1)$ \\
\hline III & $4(8.2)$ \\
\hline IV & $13(26.5)$ \\
\hline I+ IV & $10(20.4)$ \\
\hline$\|+\|$ & $8(16.3)$ \\
\hline III + IV & $4(8.2)$ \\
\hline Giant & $5(10.2)$ \\
\hline Recurrence w/in new insular zone, $\mathrm{n}(\%)$ & $17(34.7)$ \\
\hline \multicolumn{2}{|l|}{ Mean preop tumor volume $\pm \mathrm{SD}, \mathrm{cm}^{3}$} \\
\hline Grade II & $20.7 \pm 19.3$ \\
\hline Grade III & $17.0 \pm 14.4$ \\
\hline Grade IV & $14.5 \pm 12.4$ \\
\hline \multicolumn{2}{|l|}{ Primary symptom at recurrence, $\mathrm{n}(\%)$} \\
\hline Asymptomatic & $35(71.4)$ \\
\hline Worsening frequency of seizures & $10(20.4)$ \\
\hline Worsening hemiparesis & $2(4.1)$ \\
\hline Headaches & $1(2.0)$ \\
\hline Facial numbness & $1(2.0)$ \\
\hline \multicolumn{2}{|l|}{ Prior adjuvant treatment, $\mathrm{n}(\%)$} \\
\hline None & $9(18.4)$ \\
\hline Chemotherapy & $23(46.9)$ \\
\hline RT & $3(6.1)$ \\
\hline Chemotherapy + RT & $14(28.6)$ \\
\hline Median follow-up after reop (range), yrs & 2.03 ( 2 days -9.19 yrs) \\
\hline
\end{tabular}

${ }^{*}$ From index surgery.

At the time of reoperation, $44.9 \%$ of previously grade II or III tumors demonstrated malignant transformation to a higher WHO grade.

\section{Surgical Approach and Treatment Details}

During reoperation, $95.9 \%$ of cases utilized language and/or motor mapping (Table 2). Median EORs of grade II,
TABLE 2. Treatment details

\begin{tabular}{lc}
\hline \multicolumn{1}{c}{ Treatment Characteristic } & Value (\%) \\
\hline Language mapping & $23(46.9)$ \\
\hline Motor mapping & $40(81.6)$ \\
\hline Awake surgery & $26(53.1)$ \\
\hline Adjuvant therapy (prior to progression) & 9 \\
\hline None & 2 \\
\hline Alone RT & 22 \\
\hline Alone chemotherapy & 13 \\
\hline RT + chemotherapy & 1 \\
\hline Convection enhanced delivery/chemotherapy & 2 \\
\hline Unknown &
\end{tabular}

III, and IV tumors were $82.1 \%, 75.0 \%$, and $94.6 \%$, respectively (Table 3 ), when analyzing the insular component only. Two cases are depicted demonstrating the degree of resection (Figs. 1 and 2). Postoperatively, the majority of patients underwent chemotherapy and/or radiation therapy $(86.4 \%)$. EOR was not significantly impacted by a number of tumor and patient factors including sex, tumor laterality, insular zone, and awake mapping (Table 4). Furthermore, age, time to reoperation, preoperative insular or total tumor volume, and percentage of tumor within the insula were not significantly associated with EOR (data not shown).

\section{Perioperative Morbidity}

No immediate postoperative complications were observed in 79.6\% $(n=39)$ of cases (Table 5). Apart from postoperative neurological deficits, the 5 other complications observed included 1 wound infection that required washout, 1 case of hyponatremia that required intervention, 1 postoperative seizure, 1 case of a deep vein thrombosis (DVT), and 1 stroke within the ipsilateral lentiform nucleus. At the time of hospital discharge, $83.7 \%$ of cases had no new or worsened neurological deficit after resection. In terms of postoperative deficits attributable to the insular region, there were 2 cases $(4.1 \%)$ of an isolated mild facial droop, 1 case $(2.0 \%)$ of hemiplegia (Medical Research Council [MRC] grade 0/5), and 1 case (2.0\%) of a hemineglect syndrome. As these tumors often extended into neighboring frontal or temporal cortex along with adjacent subcortical language tracts, deficits related to these structures were also observed. This included 3 patients (6.1\%) who developed mild word-finding difficulty and 3 (6.1\%) who developed a new or worsened visual field cut postoperatively.

Ninety-day postoperative assessment $(n=47)$ demon-

TABLE 3. Extent of resection according to WHO grade

\begin{tabular}{cc}
\hline Grade & Median EOR \pm SD, $\%$ \\
\hline II & $82.1 \pm 16.5$ \\
\hline III & $75.0 \pm 18.6$ \\
\hline IV & $94.6 \pm 20.3$ \\
\hline
\end{tabular}



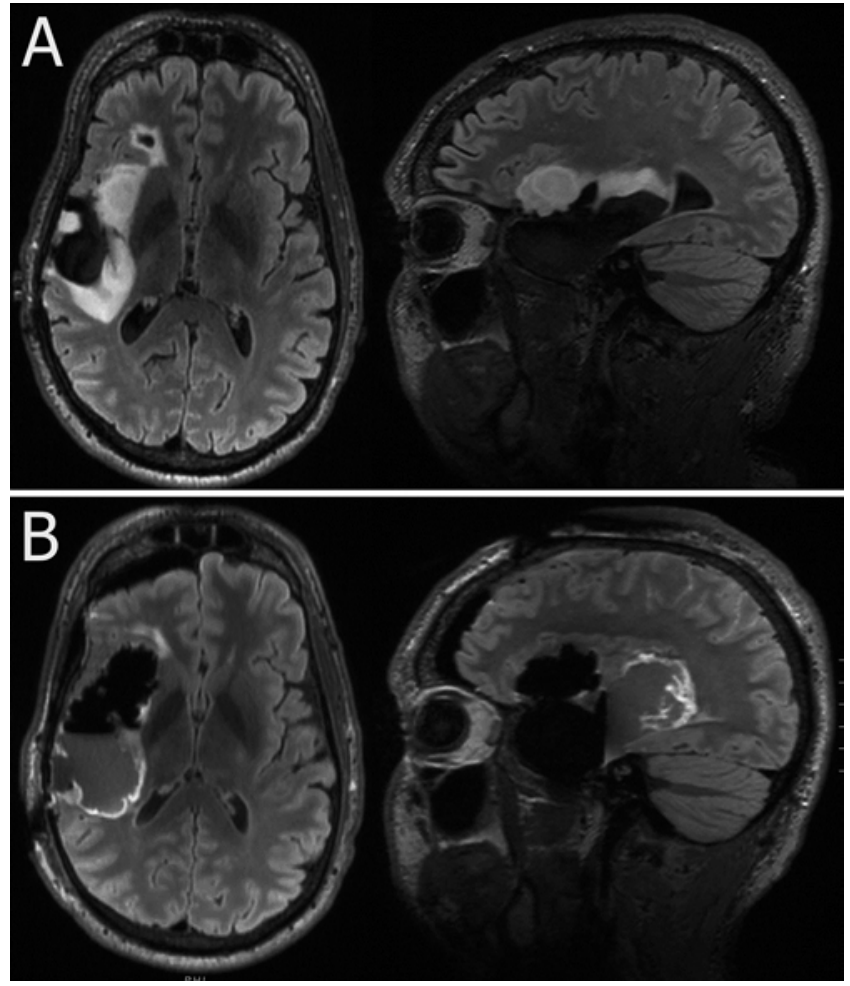

FIG. 1. Preoperative (A) and postoperative (B) axial and sagittal MR FLAIR images from the case of a 42-year-old man who had previously undergone two resections of a grade II oligoastrocytoma and subsequently underwent repeat resection after presenting with gradual tumor progression on imaging. Pathology demonstrated a grade II diffuse glioma with $95 \%$ of the insular component resected during the operation.

strated that $91.5 \%$ of patients had no postoperative deficit. Of the patients with a deficit at 90 days, $3(6.4 \%)$ had a visual field cut, and 1 (2.1\%) had hemiparesis (MRC grade 1/5) attributable to a pericavity infarct as seen on DWI noted initially postoperatively. Of note, the patient with persistent hemiparesis had a glioblastoma that progressed rapidly within 6 weeks of surgery, which prevented further recovery of the dense hemiparesis observed initially after surgery. All visual field cuts observed were persistent on follow-up, but all patients with word-finding difficulty had symptomatic resolution by the end of follow-up.

The presence of a new or worsened postoperative deficit at discharge or 90 days was not significantly impacted by age, insular zone, tumor side, awake mapping, time to reoperation, or preoperative insular or total tumor volume (Table 6). However, gadolinium-enhancing masses, larger preoperative total volume but not preoperative insular volume was predictive of a persistent postoperative deficit ( $\mathrm{p}$ $=0.0242$ ). Additionally, patients with a persistent postoperative deficit and an enhancing tumor were found to have a lower EOR ( $p=0.0158)$ suggesting that more difficult, higher-grade lesions that could not be removed completely were also associated with a greater risk of postoperative deficit.

\section{Discussion}

Insular gliomas remain challenging lesions to resect,
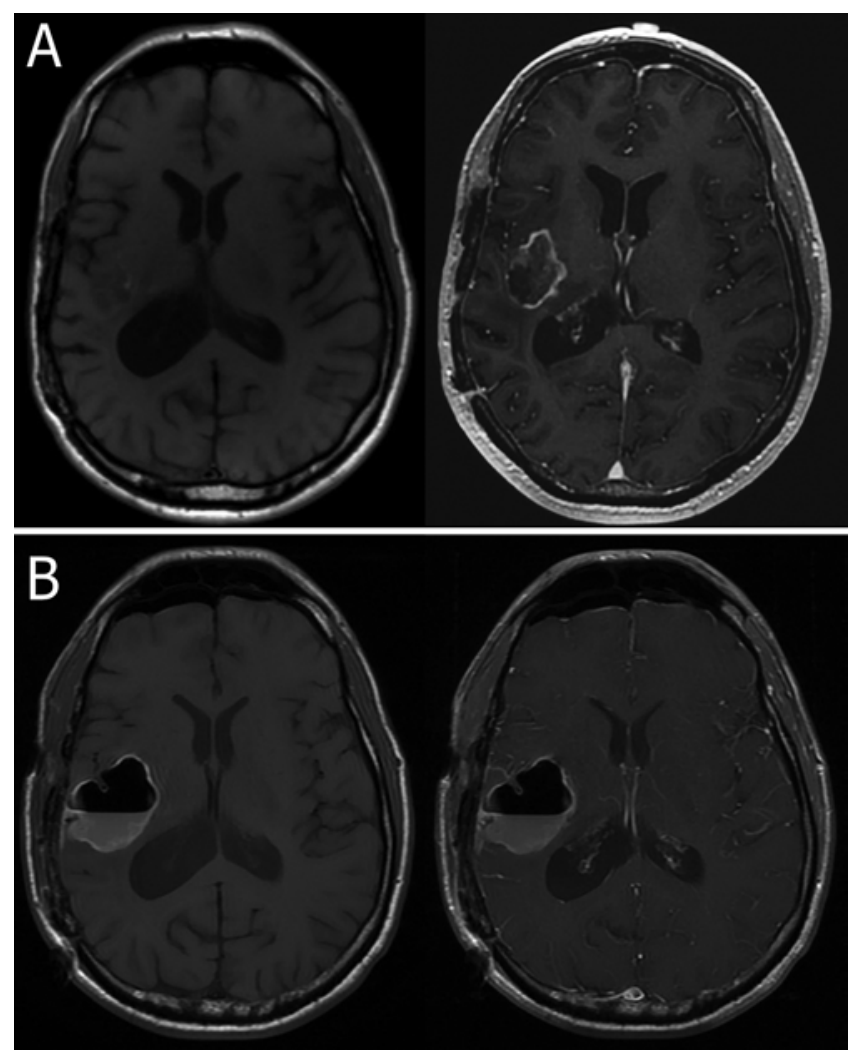

FIG. 2. Preoperative (A) and postoperative (B) axial T1-weighted MR images without and with contrast from the case of a 47-year-old man who had previously undergone two resections of a grade II oligoastrocytoma and subsequently underwent repeat resection after presenting a new contrast-enhancing portion on imaging. Pathology demonstrated a grade III anaplastic oligodendroglioma with $100 \%$ of the contrastenhancing insular component resected during the operation.

especially upon reoperation. Anatomically, these lesions are in close proximity to important vascular structures including middle cerebral artery (MCA) branches and lenticulostriate vessels. Furthermore, primary motor and sensory areas and the perisylvian language network are adjacent to these tumors and at risk of mechanical or ischemic injury intraoperatively. Some authors believe that the risk of vascular injury is much lower upon reoperation due to the prior dissection of the MCA and its branches at the time of primary surgery. ${ }^{6}$ However, others believe that adhesions, gliosis, and recurrent tumor tissue may obscure previously exposed vessels and disrupt anatomical landmarks. Gliosis and adhesions may also pose a risk to underlying functional tissue. Upon opening dura, for example, excess tension via adhesions underlying the dura may disrupt nearby cortex. Prior reports also state that tumor recurrence in this region often manifests as tumor expansion into an empty resection cavity, implying that tumors often do not recur outside their initial insular zone. ${ }^{6}$ While this may be the case in some patients, we have observed numerous instances of new invasion into neighboring insular zones with $34.7 \%$ of tumors recurring within a new insular zone. Reoperation can, however, be facilitated by the prior resection cavity that often provides a clear operative corridor to approach recurrent gliomas. 
TABLE 4. Tumor and patient factors versus EOR

\begin{tabular}{ccc}
\hline \multicolumn{1}{c}{ Factor } & Mean $\%$ EOR \pm SD & p Value \\
\hline Sex & & 0.45 \\
\hline Male & $78.1 \pm 3.2$ & \\
\hline Female & $82.2 \pm 4.3$ & 0.56 \\
\hline Laterality & & \\
\hline Lt & $78.0 \pm 3.7$ & 0.57 \\
\hline Rt & $81.0 \pm 3.6$ & \\
\hline Mapping & & \\
\hline Awake & $78.2 \pm 3.5$ & \\
\hline Asleep & $81.1 \pm 3.7$ & \\
\hline Berger-Sanai zone & & \\
\hline I & $93.3 \pm 12.4$ & \\
\hline II & $94.0 \pm 10.1$ & \\
\hline III & $84.1 \pm 8.8$ & \\
\hline IV & $82.7 \pm 4.9$ & \\
\hline 2 quadrants & $73.4 \pm 3.7$ & \\
\hline Giant & $80.4 \pm 7.8$ & \\
\hline
\end{tabular}

As prior studies, focusing primarily on newly diagnosed insular gliomas, demonstrate that median EOR of $80 \%-82 \%$ is associated with low perioperative and longterm morbidity and a survival benefit regardless of location, it is important to provide evidence of similar findings for recurrent gliomas in this region. ${ }^{3,4,13,14}$ However, outcomes after reoperation for recurrent gliomas within the insula have been underreported. With this in mind, our goal was to determine the morbidity and the EOR achievable in addition to technical considerations during reoperation.

Historically, some surgeons speculated that infiltration into functional tissue combined with nearby vascular structures made the risk of a permanent deficit due to stroke or violation of functional tissue too great to attempt repeat operation for insular gliomas. ${ }^{6,9}$ Evidence from this study, in addition to prior studies, does not support this contention. Ius and colleagues ${ }^{6}$ reported outcomes for 23 patients with previously diagnosed insular low-grade gliomas who underwent repeat operations. Focusing on low-grade glioma, all cases involved subcortical mapping, and median EOR for tumor recurrence was reported to be $82 \%$, similar to our study. In terms of morbidity, the group reported new neurological deficits in $34.78 \%$ of patients $(17.39 \%$ motor, $13.04 \%$ speech, and $4.35 \%$ visual field deficit). At 6 months, however, neurological deficits were noted in only 1 patient (4.35\%). ${ }^{6}$ Martino and colleagues examined outcomes in 19 patients undergoing repeat resection of tumors in eloquent cortex, with 3 of these patients harboring tumors within the insula. With the use of cortical mapping techniques, all 3 cases were associated with partial or subtotal resection. ${ }^{9}$ Our results, which include both low- and high-grade gliomas, demonstrate that $91.5 \%$ of patients undergoing reoperation had no new or worsened postoperative deficit at 90-day followup. Additionally, although a rare complication in our study occurring only once, the most marked morbidity was sec-
TABLE 5. Outcomes

\begin{tabular}{lc}
\hline \multicolumn{1}{c}{ Outcome } & Value $(\%)$ \\
\hline No complications & $39(79.6)$ \\
\hline Postop complications $^{*}$ & $8(16.3)$ \\
\hline New neurological deficit & $1(2.04)$ \\
\hline Wound infection & $1(2.04)$ \\
\hline Hyponatremia & $1(2.04)$ \\
\hline Postop seizures & $1(2.04)$ \\
\hline DVT & $1(2.04)$ \\
\hline Infarct & \\
\hline Neurological deficits at discharge & $41(83.7)$ \\
\hline None & $3(6.1)$ \\
\hline Mild word-finding difficulty (names $>50 \%)$ & $2(4.1)$ \\
\hline Mild facial droop & $1(2)$ \\
\hline Hemiplegia & $1(2)$ \\
\hline Hemineglect & $3(6.1)$ \\
\hline Visual field cut & \\
\hline Neurological deficits at 90 days $\dagger$ & $43(91.5)$ \\
\hline None & $3(6.4)$ \\
\hline Visual field cut & $1(2.1)$ \\
\hline Hemiparesis (strength grade 1-2/5)
\end{tabular}

* Patients may have $\geq 1$ complication.

† Two patients were lost to follow-up and 1 patient had rapid tumor progression with associated new neurological deficits (hemiparesis) within 3 months of the operation.

ondary to an infarct, which emphasizes the importance of identifying vascular structures during resection, especially when adhesions are present. Yet, even if vascular structures are carefully protected, the occurrence of ischemic injury may be difficult to prevent. Indeed, we typically apply small pieces of papaverine-soaked Gelfoam to exposed arteries to prevent vasospasm-related ischemia, which did not appear to prevent stroke in this particular patient. While our total rate of postoperative deficits on follow-up was slightly higher than previously reported, this included visual field deficits that were likely due to resection of components of the tumor extending into visual pathways of the temporal lobe and not within the confines of the insular region.

In terms of morbidity, reoperation on insular tumors has similar rates of postoperative deficits in both the short- and long-term when compared to newly diagnosed gliomas. Our prior reports demonstrate a rate of long-term (90-day) motor or language deficits in $3.2 \%-5.8 \%$ of patients. ${ }^{5,13}$ Skrap and colleagues reported a median EOR of $80 \%$ in nonenhancing newly resected insular gliomas and found that about one-third of patients had an immediate postoperative speech or motor deficit, with $95 \%$ of patients returning to their neurological baseline at 6 months. $.^{14} \mathrm{Me}-$ dian EOR rates for insular tumor reoperation also closely match those seen for newly treated tumors, which have been reported to be $80 \%-85 \%$. $5,13,14$

Patient selection for reoperation is critical, and predictors for benefit from reoperation in prior studies have included Karnofsky Performance Scale score $>70$, age $<50$ 
TABLE 6. Tumor and patient factors versus deficits at discharge and by 90 days' follow-up

\begin{tabular}{|c|c|c|c|c|c|c|}
\hline \multirow[b]{2}{*}{ Factor } & \multicolumn{3}{|c|}{ Deficits at Discharge } & \multicolumn{3}{|c|}{ Deficits at 90 Days $^{*}$} \\
\hline & Yes & No & $\mathrm{p}$ Value & Yes & No & $\mathrm{p}$ Value \\
\hline Mean age $\pm S D$, yrs & $43.7 \pm 3.3$ & $43.7 \pm 3.3$ & 0.53 & $42.0 \pm 4.8$ & $41.8 \pm 1.5$ & 0.98 \\
\hline Lt laterality, n (\%) & $3(6.1)$ & $21(42.9)$ & 0.48 & $1(2.1)$ & $22(46.8)$ & 0.31 \\
\hline Awake mapping, $n(\%)$ & $5(10.2)$ & $21(42.9)$ & 0.56 & $2(4.3)$ & $23(48.9)$ & 0.89 \\
\hline Mean time to reop, yrs & 4.4 & 4.5 & 0.88 & $3.0 \pm 1.5$ & $4.6 \pm 0.5$ & 0.31 \\
\hline Mean preop insular FLAIR volume $\pm \mathrm{SD}, \mathrm{cm}^{3}$ & $18.4 \pm 2.4$ & $23.2 \pm 3.5$ & 0.58 & $22.7 \pm 11.5$ & $22.5 \pm 3.5$ & 0.99 \\
\hline Mean preop total FLAIR volume \pm SD, $\mathrm{cm}^{3}$ & $66.0 \pm 18.4$ & $55.3 \pm 8.1$ & 0.60 & $80.4 \pm 26.4$ & $55.1 \pm 8.1$ & 0.37 \\
\hline Mean $\%$ EOR \pm SD & $78.5 \pm 6.4$ & $79.7 \pm 2.8$ & 0.86 & $78.2 \pm 8.7$ & $80.0 \pm 2.7$ & 0.84 \\
\hline Berger-Sanai zone, n (\%) & & & 0.74 & & & 0.67 \\
\hline I & $0(0)$ & $2(4.1)$ & & $0(0)$ & $2(4.3)$ & \\
\hline II & $1(2.0)$ & $2(4.1)$ & & $0(0)$ & $3(6.4)$ & \\
\hline III & $0(0)$ & $4(8.2)$ & & $0(0)$ & $4(8.5)$ & \\
\hline IV & $2(4.1)$ & $11(22.5)$ & & $2(4.3)$ & $11(23.4)$ & \\
\hline 2 quadrants & $4(8.2)$ & $18(36.7)$ & & $2(4.3)$ & $18(38.3)$ & \\
\hline Giant & $1(2.0)$ & $4(8.2)$ & & $0(0)$ & $5(10.6)$ & \\
\hline
\end{tabular}

* Data not available for 2 patients.

years, greater initial EOR, and longer interval between operations. ${ }^{4}$ These data suggest that insular location can and should be considered for reoperation at recurrence. Even if tumor recurrence is observed in areas of previously noted functional tissue (i.e., at the time of prior mapping of these regions during index surgery), this is not necessarily a contraindication to reoperation as reorganization of functional networks may make these areas accessible. Prior reports examining glioma reoperations found reorganization of motor and language areas at the time of second surgery at a rate of $30 \% .{ }^{15}$ Such reorganization often allows for further resection of tumor tissue upon reoperation. .15

These results add to our understanding of the operative management of insular gliomas in several ways. First, we report on specific outcomes in the largest cohort of recurrent low- and high-grade insular glioma patients undergoing reoperation to date. Unlike with newly diagnosed insular gliomas, we did not find a significant effect of insular zone classification on EOR or frequency of postoperative deficits. One reason for this may be that operative corridors created by the first operation allow easier access to the site of recurrence, making approaches to the various zones similar in technical difficulty. Of note, EOR did not significantly vary based on insular tumor size, suggesting that even larger recurrences may be amenable to extensive resection. There are limitations associated with this study due to its retrospective nature. In addition, although this is the largest reported cohort of patients with insular glioma, we did not report the impact of EOR on survival because this was not the goal of conducting the study. Furthermore, although we report a low morbidity rate, the results of this single-center study may not be generalizable to other clinical settings.

\section{Conclusions}

This study demonstrates that recurrent insular gliomas of all degrees of insular involvement and tumor grade can be safely and extensively resected. Therefore, reoperation should be considered and offered to patients when recurrence is identified as part of the management plan.

\section{Acknowledgments}

We would like to thank Jing Li for completing the search of the institutional database for patients qualifying for this study.

\section{References}

1. Benet A, Hervey-Jumper SL, Sánchez JJ, Lawton MT, Berger MS: Surgical assessment of the insula. Part 1: surgical anatomy and morphometric analysis of the transsylvian and transcortical approaches to the insula. J Neurosurg 124:469-481, 2016

2. Bloch O, Han SJ, Cha S, Sun MZ, Aghi MK, McDermott MW, et al: Impact of extent of resection for recurrent glioblastoma on overall survival: clinical article. J Neurosurg 117:1032-1038, 2012

3. Duffau H, Moritz-Gasser S, Gatignol P: Functional outcome after language mapping for insular World Health Organization Grade II gliomas in the dominant hemisphere: experience with 24 patients. Neurosurg Focus 27(2):E7, 2009

4. Hervey-Jumper SL, Berger MS: Reoperation for recurrent high-grade glioma: a current perspective of the literature. Neurosurgery 75:491-499, 2014

5. Hervey-Jumper SL, Li J, Osorio JA, Lau D, Molinaro AM, Benet A, et al: Surgical assessment of the insula. Part 2: validation of the Berger-Sanai zone classification system for predicting extent of glioma resection. J Neurosurg 124:482488, 2016

6. Ius T, Pauletto G, Cesselli D, Isola M, Turella L, Budai R, et al: Second surgery in insular low-grade gliomas. Biomed Res Int 2015:497610, 2015

7. Ius T, Pauletto G, Isola M, Gregoraci G, Budai R, Lettieri $\mathrm{C}$, et al: Surgery for insular low-grade glioma: predictors of postoperative seizure outcome. J Neurosurg 120:12-23, 2014

8. Lang FF, Olansen NE, DeMonte F, Gokaslan ZL, Holland EC, Kalhorn C, et al: Surgical resection of intrinsic insular tumors: complication avoidance. J Neurosurg 95:638-650, 2001 
9. Martino J, Taillandier L, Moritz-Gasser S, Gatignol P, Duffau $\mathrm{H}$ : Re-operation is a safe and effective therapeutic strategy in recurrent WHO grade II gliomas within eloquent areas. Acta Neurochir (Wien) 151:427-436, 2009

10. Moshel YA, Marcus JD, Parker EC, Kelly PJ: Resection of insular gliomas: the importance of lenticulostriate artery position. J Neurosurg 109:825-834, 2008

11. Pallud J, Audureau E, Blonski M, Sanai N, Bauchet L, Fontaine D, et al: Epileptic seizures in diffuse low-grade gliomas in adults. Brain 137:449-462, 2014

12. Ringel F, Pape H, Sabel M, Krex D, Bock HC, Misch M, et al: Clinical benefit from resection of recurrent glioblastomas: results of a multicenter study including 503 patients with recurrent glioblastomas undergoing surgical resection. Neuro Oncol 18:96-104, 2016

13. Sanai N, Polley MY, Berger MS: Insular glioma resection: assessment of patient morbidity, survival, and tumor progression. J Neurosurg 112:1-9, 2010

14. Skrap M, Mondani M, Tomasino B, Weis L, Budai R, Pauletto G, et al: Surgery of insular nonenhancing gliomas: volumetric analysis of tumoral resection, clinical outcome, and survival in a consecutive series of 66 cases. Neurosurgery 70:1081-1094, 2012

15. Southwell DG, Hervey-Jumper SL, Perry DW, Berger MS: Intraoperative mapping during repeat awake craniotomy reveals the functional plasticity of adult cortex. J Neurosurg 124:1460-1469, 2016

16. Sughrue ME, Sheean T, Bonney PA, Maurer AJ, Teo C: Aggressive repeat surgery for focally recurrent primary glioblastoma: outcomes and theoretical framework. Neurosurg Focus 38(3):E11, 2015

17. Uppstrom TJ, Singh R, Hadjigeorgiou GF, Magge R, Rama- krishna R: Repeat surgery for recurrent low-grade gliomas should be standard of care. Clin Neurol Neurosurg 151:1823, 2016

18. Wang DD, Deng H, Hervey-Jumper SL, Molinaro AA, Chang EF, Berger MS: Seizure outcome after surgical resection of insular glioma. Neurosurgery [epub ahead of print], 2017

19. Wu AS, Witgert ME, Lang FF, Xiao L, Bekele BN, Meyers CA, et al: Neurocognitive function before and after surgery for insular gliomas. J Neurosurg 115:1115-1125, 2011

\section{Disclosures}

The authors report no conflict of interest concerning the materials or methods used in this study or the findings specified in this paper.

\section{Author Contributions}

Conception and design: Berger. Acquisition of data: all authors. Analysis and interpretation of data: Berger, Morshed, Han, Hervey-Jumper. Drafting the article: all authors. Critically revising the article: all authors. Reviewed submitted version of manuscript: all authors. Approved the final version of the manuscript on behalf of all authors: Berger. Statistical analysis: Morshed. Administrative/technical/material support: Morshed, HerveyJumper. Study supervision: Berger, Morshed, Hervey-Jumper.

\section{Correspondence}

Mitchel S. Berger: University of California, San Francisco, CA. mitchel.berger@ucsf.edu. 